Effect of MIL-53 (Al) MOF particles on the chain mobility and crystallization of poly(L-lactic acid)

Peer-reviewed author version

Kathuria, Ajay; Brouwers, Niels; BUNTINX, Mieke; Harding, Trevor \& Auras, Rafael (2017) Effect of MIL-53 (Al) MOF particles on the chain mobility and crystallization of poly(L-lactic acid). In: JOURNAL OF APPLIED POLYMER SCIENCE, 134, p. 1-8 (Art N 45690).

DOI: 10.1002/APP.45690

Handle: http://hdl.handle.net/1942/24427 


\section{Effect of MIL-53 (Al) MOF particles on the chain mobility and crystallization of Poly(L-lactic acid)}

3 Ajay Kathuria ${ }^{1 *}$, Niels Brouwers ${ }^{1,2}$, Mieke Buntinx ${ }^{2}$, Trevor Harding ${ }^{3}$, Rafael Auras ${ }^{4}$

$4 \quad{ }^{1}$ Industrial Technology and Packaging, California Polytechnic State University, San Luis 5 Obispo, CA 93407, USA

$6 \quad 2$ Packaging Technology Center, IMO-IMOMEC, Hasselt University, 3590 Diepenbeek, 7 Belgium

$8 \quad{ }^{3}$ Materials Engineering, California Polytechnic State University, San Luis Obispo, CA 993407 , USA

$10{ }^{4}$ School of Packaging, Michigan State University, East Lansing, MI, USA 48824-1223

$12 *$ Corresponding author:

13 P: (805) 756-2944; F: (805) 756-6111; E: akathuri@ calpoly.edu

14

15 Accepted manuscript Journal of Applied Polymer Science 


\section{Abstract}

17 Polymer-filler interactions significantly influence morphology, functionality and various

18 desirable properties of mixed matrix membranes (MMMs). In this study, chain mobility

19 and crystallization of Poly(L-lactic acid) (PLLA) MMM films prepared by solvent casting

20 PLLA with 1, 5, 10 and 20\% wt./wt. of MIL-53(Al) metal organic framework (MOF) were

21 evaluated. The fabricated MMMs were characterized using Differential Scanning

22 Calorimetry (DSC), Fourier Transform Infrared Spectroscopy (FTIR), Thermogravimetric

23 Analysis (TGA), and Scanning Electron Microscopy (SEM). DSC studies indicated that

24 the addition of MOF particles in the PLLA matrix reduces the polymeric chain mobility,

25 which affects the crystallization process. The percent crystallinity of neat PLLA was found

26 to decrease from $4 \%$ in neat PLLA to completely amorphous structures in PLLA-10\% and

27 PLLA-20\% MMMs, as observed in the second heating cycle. FTIR data supports these

28 observations. TGA results showed that PLLA-MOF films are thermally less stable than

29 neat PLLA suggesting that MOF particles act as a depolymerization catalyst for PLLA.

30 Partial agglomeration of MOF particles was observed in the samples using SEM studies.

31 This study indicates strong PLLA-MIL-53(Al) MOF interactions. In addition, this study

32 also provides insight into the effect of MOF particles on the segmental mobility and 33 morphology of PLLA-MIL-53 (Al) composite films. 


\section{Introduction}

37 Fillers, functional materials, additives or modifiers have been widely used to improve the

38 functionality of polymeric matrices, including strength, stiffness, hardness, durability,

39 permeability and controlled selective exchange of gases for various applications [1-3].

40 Interfacial interactions between the matrix and filler play an influential role in polymer-

41 filler dynamics. Surface area, concentration and particle size of the filler influence the

42 polymeric chain structure, segmental mobility and crystallinity [4-5]. The interfacial region

43 and its surroundings behave differently from the bulk polymeric matrix. Good polymer-

44 filler interactions can create topological restraints, which may hinder the mobility of

45 polymeric chains. On the other hand, poor interfacial interactions lead to interfacial voids

46 or defects $[1,3,5-8]$.

47 Poly(lactic acid) (PLA) is a bio-based, bio-degradable and compostable polymer

48 that has consolidated its market presence due to techno-commercial advancement, growing

49 production capacity and economic viability [9-10]. PLA is a clear, rigid thermoplastic with

50 mechanical strength comparable to poly(ethylene terephthalate) (PET) and polystyrene

51 (PS). However, various inherent limitations such as its brittle nature, poor barrier

52 performance and slow crystallization kinetics limit its wide scale commercial use [1].

53 Metal-organic frameworks (MOFs) belongs to a class of microporous, high surface

54 area crystalline materials synthesized by coordinating metal ions with organic struts [11].

55 Their varied synthesis techniques and potential application in areas such as catalysis, gas

56 storage, gas separation, selectivity, chemical sensing, encapsulation and drug delivery have

57 been comprehensively reported by researchers in the last two decades [11-14]. These 
reticulate symmetrical crystals can be designed with specific structural properties to meet

59 desired objectives among other organic, inorganic and hybrid porous structures [15-17].

MIL-53 (Al) is a highly flexible, thermally and structurally stable, hydrolysis

61 resistant commercially available microporous MOF which is formed by coordination of

62 trivalent aluminum with benzene dicarboxylate struts. It is known for its high surface area,

63 high $\mathrm{CO}_{2}$ adsorption and selectivity [18, 19]. Ferey et al. [20] reported that MIL-53 (Al)

64 has high sorption and selectivity for $\mathrm{CO}_{2}$ gas over other gases such as $\mathrm{O}_{2}$ and $\mathrm{CH}_{4}$. In

65 addition, MIL-53 (Al) is known for its distinct breathing attribute due to the reversible pore

66 expansion and contraction behavior under varying sorbent pressure [21].

67 Ease of processibility and cost effectiveness of the polymers along with high

68 selectivity of MOF particles motivates fabrication of polymer-MOF mixed matrix

69 membranes (MMMs). The polymer-MOF interfacial interaction, polymeric chain mobility,

70 crystallization kinetics and percentage crystallinity of a polymer significantly influences

71 its performance and functionality. Traditionally, interfacial studies have attracted attention

72 in order to achieve higher stress transfer for superior mechanical performance.

73 Additionally, poor interfacial adhesion, or the presence of defects, affects various

74 important properties including gas diffusion and permeability. In the case of polymer-MOF

75 composites, five types of interfacial morphologies have been reported depending on the

76 interfacial interactions are: a). two phase defect free, b). poor interactions leading to voids

77 c). dilated d). increased density e). plugged morphology which can significantly impact

78 their transmission, permeability and perm-selectivity [22]. Such selectively permeable

79 polymer-membranes system can be tailored to gas composition in the headspace of a 
80 product-package system to optimize the shelf life of various consumer products including

81 respiring product such as fresh produce, cheese etc.

82 Chain mobility is of great significance during the final stages of polymer

83 processing. In addition, segmental chain mobility can influence the physical aging of

84 polymeric materials, which can alter functional properties particularly in glassy polymers

85 including permeability and selectivity [23-24]. Poly (L-lactic acid) (PLLA), a low

86 entanglement density polymer, has demonstrated further decrease in the entanglement

87 density with physical aging due to segmental mobility of the chains [23]. Such

88 microstructural changes can adversely affect various functional properties including

89 mechanical performance, gas transportation and transparency. Previous studies have linked

90 reduced segmental mobility of polymer-nanocomposites with strong interfacial

91 interactions [1, 24-25]. Segmental mobility of PLA chains is of profound interest as it

92 influences crystallinity, as well as its mechanical, barrier and other functional properties

$93[7-8,26]$.The purpose of this study was to evaluate the effect of MIL-53 (Al) MOF particles

94 on the segmental mobility and crystallization of solvent cast PLLA films. The effect of

95 varying MOFs fraction on the PLLA matrix was analyzed by using an array of 96 characterization techniques.

\section{2. Methodology}

\section{$98 \quad 2.1$ Materials}

99 Poly(L-lactic acid) (PLLA) resin grade 4043 D, (98\% L-lactide) pellets were supplied by

100 NatureWorks LLC (Blair, NE, USA). The weight average molecular weight $\left(M_{w}\right)$ was 111

$101 \mathrm{kDa}$, with a number average molecular weight $\left(M_{n}\right)$ of $84 \mathrm{kDa}$ and a polydispersity index 
$102\left(M_{w} / M_{n}\right)$ of 1.3. MIL-53 (Al) MOF produced by BASF® was acquired from Sigma-Aldrich

103 (St. Louis, MO, USA) under the trade name of Basolite ${ }^{\mathrm{TM}} \mathrm{A} 100 \mathrm{MOF}\left(\mathrm{C}_{8} \mathrm{H}_{5} \mathrm{AlO}_{5}\right)$, with a

104 surface area between 1,100 and $1,500 \mathrm{~m}^{2} \mathrm{~g}^{-1}$ and particle size distribution of $31.55 \mu \mathrm{m}$.

105 Chloroform [anhydrous $\geq 99 \%$ ] was purchased from Sigma-Aldrich, St. Louis, MO, USA.

$106 \quad 2.2$ Methods

107 PLLA pellets were dried at $80{ }^{\circ} \mathrm{C}$ for $4 \mathrm{~h}$ using a Thermo Fisher Scientific VWR Oven 108 (Fisher Scientific, Waltham, MA, USA) with a negative pressure of $2.93 \mathrm{kPa}$ and packed 109 in an air tight glass bottle. Three grams of dried PLLA pellets were gradually poured into

$11075 \mathrm{~mL}$ of chloroform at $23^{\circ} \mathrm{C}$ while mixing the solution with a magnetic stir plate (Fisher

111 Scientific, Waltham, MA, USA) at 300 RPM for approximately 90 min until all PLLA

112 pellets were dissolved. Basolite ${ }^{\mathrm{TM}}$ A100 MOF $\left(\mathrm{C}_{8} \mathrm{H}_{5} \mathrm{AlO}_{5}\right)$ particles were crushed by

113 applying light, concentric pressure using a Green Marble Mortar \& Pestle. PLLA, PLLA-

$1141 \%$ MOF, PLLA-5\% MOF, PLLA-10\% MOF and PLLA-20\% MOF were processed by

115 solvent casting. The desired MOF content was weighed using a Mettler Toledo Scale,

116 Model ME54E (Columbus, OH, USA) and poured in the dissolved PLLA-chloroform

117 solution while mixing the solution with the magnetic stir plate rotating at 300 RPM for 10

118 s. Ultrasonication was performed on the PLLA-MOF-chloroform solution using a Q500

119 ultrasonicator purchased from QSonica, LLC (Newtown, CT, USA). An on-off cycle was

120 programmed to ultrasonicate for $3 \mathrm{~min}$, with an on cycle time of $3 \mathrm{~s}$ at a frequency of 20

$121 \mathrm{kHz}$ and an alternating off cycle of $0 \mathrm{~Hz}$ for $2 \mathrm{~s}$. The ultrasonicated solution was poured

122 into teflon coated molds and covered with two layers of holed Al foil to control the

123 chloroform evaporation rate. After completely evaporating the chloroform, the solid

124 samples were placed in the vacuum oven at $23{ }^{\circ} \mathrm{C}$ and a negative pressure of $22 \mathrm{mmHg}$ to 
extract the residual chloroform from the samples. Samples were stored in vacuum bags at room temperature until the beginning of the different tests. The resulting films had an

127 average thickness of $178 \pm 25 \mu \mathrm{m}(7.00 \pm 1.0 \mathrm{mil})$.

$128 \quad 2.3$ Differential Scanning Calorimetry (DSC)

129 DSC analysis of neat PLLA and PLLA-MIL 53 (Al) MMM films was performed using a 130 DSC Q1000 (TA instruments, DE, USA). Samples size between 5 and $10 \mathrm{mg}$ were 131 obtained from the films and non-hermetically sealed in an aluminum pan and placed with 132 the reference pan in the DSC cell and run in triplicates. Heat/cool/heat cycles from $0{ }^{\circ} \mathrm{C}$ to $133180{ }^{\circ} \mathrm{C}$ at a rate of $10{ }^{\circ} \mathrm{C} \min ^{-1}$ were performed under a constant nitrogen flow of 50 $134 \mathrm{~mL} / \mathrm{min}$. To disregard the thermal history during processing or storage, the second heating 135 cycle was used to determine the glass transition temperature $\left(T_{g}\right)$, cold crystallization onset $136\left(T_{c o}\right)$, melting temperature $\left(T_{m}\right)$ and to calculate the enthalpy of cold crystallization $\left(\Delta H_{c}\right)$ 137 and fusion $\left(\Delta H_{m}\right)$. The percent crystallinity $X c(\%)$ was estimated using the following 138 equation:

$$
X_{c}(\%)=\frac{\Delta H_{m}-\Delta H_{c}}{\Delta H_{m}^{c}(1-x)} \times 100
$$

140 where $\Delta H_{m}$ is enthalpy of fusion; $\Delta H_{c}$ is the enthalpy of cold crystallization; $\Delta H^{c}{ }_{m}$ is 141 enthalpy of fusion of pure crystalline PLA $\left(\Delta H^{c}{ }_{m}=93.1 \mathrm{~J} / \mathrm{g}\right.$ [27] $)$ and $x$ represents the mass 142 fraction of the MOF in the films. Universal Analysis software version 2000 (TA 143 Instruments, DE, USA) was used to analyze the data. Samples were run in triplicates.

\section{$144 \quad 2.4$ Fourier Transform Infrared Spectroscopy (FTIR)}

145 Fourier Transform Infrared Spectroscopy (FTIR) of MIL 53 (Al) MOF, cast PLLA and 146 PLLA-MIL-53 (Al) MMM films was performed using an attenuated total reflectance 
147 (ATR) module attached to FTIR model IRaffiny-1S (Shimadzu, CA, USA). The spectra

148 were acquired in absorption mode at room temperature in the range of $4000-400 \mathrm{~cm}^{-1}$ at 2

$149 \mathrm{~cm}^{-1}$ resolution using 40 accumulated scans. The background spectra used for reduction

150 were collected at room temperature and samples were examined in triplicates.

$151 \quad 2.5$ Thermogravimetric Analysis (TGA)

152 Thermogravimetric analyses of MIL-53 Al-MOF, neat PLLA and PLLA-MOF films were

153 investigated using a TGA Q50 (TA instruments, DE, USA) under sample nitrogen flow of

$15460 \mathrm{~mL} \mathrm{~min}^{-1}$ and balance purge flow of $40 \mathrm{~mL} \mathrm{~min}^{-1}$. Samples between 5 and $10 \mathrm{mg}$ were

155 obtained from the films and were placed in an aluminum pan before being heated at a rate

156 of $10{ }^{\circ} \mathrm{C} \mathrm{min}^{-1}$ from room temperature to $600{ }^{\circ} \mathrm{C}$. Universal Analysis software version 2000

157 (TA Instruments) was used to analyze the data and samples were run in triplicates.

$158 \quad 2.6$ Scanning Electron Microscopy (SEM)

159 Morphological analysis was performed using a Philips Quanta 200 Environmental

160 Scanning Electron Microscope (FEI, Hillsboro, OR, USA) in low vacuum mode (100 Pa)

161 with the use of a tungsten filament. SEM micrographs of the MOF powder, surfaces of

162 solvent cast PLLA and PLLA-MIL-53 (Al) MMM films were obtained at an accelerating

163 voltage of $12.5 \mathrm{kV}$.

164 2.6 Oxygen Gas Permeability Coefficient

165 The oxygen transmission rates of the PLLA and PLLA-20\% MOF were measured as per 166 ASTM D3985-05 using an Oxtran ${ }^{\circledR} 2 / 21$ (MOCON Inc., Minneapolis, MN, USA) at $23{ }^{\circ} \mathrm{C}$

167 and $0 \% \mathrm{RH}$ using $100 \%$ oxygen gas as permeant and $98 \% \mathrm{~N}_{2} / 2 \% \mathrm{H}_{2}$ as the carrier gas at 168 a flow rate of $20 \mathrm{sccm}$ of the test gas and $10 \mathrm{sccm}$ flow rate of carrier gas. The data was 169 collected every $30 \mathrm{~min}$. until stable saturated state was achieved. The transmission rate of 
170 4-6 mil thick films was measured using aluminum masks with $3.14 \mathrm{~cm}^{2}$ area. The average

171 value of the last ten stable points was used to calculate the permeability values. The data

172 were analyzed for at least three samples.

$173 \quad 2.7$ Data Analysis

174 Data analysis was carried out using SAS 9.4 Software (SAS Institute Inc., Cary, NC, USA).

175 Analysis of Variance (ANOVA) was evaluated using Tukey's HSD (Honestly Significant

176 Differences) at 95\% confidence interval $(\alpha=0.05)$.

\section{3. Results and discussion}

\section{$178 \quad 3.1$ Differential Scanning Calorimetry}

179 DSC thermograms of PLLA and PLLA-MIL-53(Al) MMMs are represented in Figure 1.

180 The onset of cold crystallization in PLLA and PLLA-1\% MOF was observed at 105.1 and

$181 \quad 108.6^{\circ} \mathrm{C}$, respectively. A shift in the onset of cold crystallization temperature of the PLLA-

$1821 \%$ MOF composition can be attributed to the good interactions between the polymeric

183 chains and the MOF crystals and high surface area of MOF hindering the segmental

184 mobility of the polymeric chains $[1,3,28]$. Such topological constraint can affect

185 crystallization kinetics and viscoelastic properties of the materials. Gagliradi et al. [28]

186 studied the interfacial interaction of poly(dimethylsiloxane) with 20 and $40 \%$ silica

187 nanoparticles and poly(vinyl acetate) with $40 \%$ silica nanoparticles. They observed

188 decreased segmental mobility, which was linked to the specific surface area (SSA) of the

189 nanoparticles, altered chain structure and changed behavior near the interfacial region

190 compared to the bulk polymeric region [28]. The extent of chain restriction also

191 sequentially increased with increase in silica nanoparticle SSA and loading in the

192 polymeric matrix. In another study, Fragiadakis et al. [29] reported higher relaxation time 
193 in the interfacial region for uniformly distributed natural rubber nano-silica particle based

194 composite systems. In this work, the absence of cold crystallization at higher MOF weight

195 fraction can be ascribed to the fact that more polymeric volume fraction was restricted due 196 to the presence of these particles.

198 Detailed analysis of the DSC thermograms of PLLA and its composites is provided 199 in Table 1 . The increase in the $T_{g}$ with increase in the MOF loading further indicates 200 reduced segmental mobility. We obtained highly amorphous neat solvent cast PLLA 201 samples with $3.2 \%$ crystallinity. The crystallinity further decreased from 3.1 to $0.5 \%$ with 2021 and 5\% MOF loading in these composites. PLLA-10\% MOF and PLLA-20\% MOF were 203 completely amorphous. At these higher MOF loadings, the cold crystallization peaks were 204 absent in the second heating cycle due to reduced chain mobility which could be ascribed 205 to strong polymer-filler interactions. The decreased percentage crystallinity with increase 206 in the MOF content can be correlated to the decreased mobility of the polymeric chains 207 [3]. Although, change in the percentage crystallinity appear to be relatively smaller 208 primarily due to amorphous nature of the polymer. The enthalpy of cold crystallization 209 represented in Table 1 provides a better picture of chain mobility. The enthalpy of chain 210 mobility of PLLA decreased from $8.7 \mathrm{~J}^{-g^{-1}}$ to $2.7 \mathrm{~J} \mathrm{~g}^{-1}$ with the addition of the $1 \%$ MIL-53 211 (Al) MOF. We did not observed any cold crystallization peaks at higher MOF loadings. 


\subsection{Thermogravimetric Analysis (TGA)}

215 Figure $2 \mathrm{a}$ and $2 \mathrm{~b}$ show TGA thermograms and derivative profiles for MOF, neat PLLA 216 and PLLA-MOF films. The first significant weight loss peak observed between 130-140 $217{ }^{\circ} \mathrm{C}$ can be ascribed to the small weight loss (2-3\%) of volatile compounds such as 218 chloroform used for solvent casting or water molecules entrapped in the plastic. Data 219 obtained from TGA thermograms is presented in Table 2. Degradation peaks were not 220 observed in MOF below $500^{\circ} \mathrm{C}$ due to its high thermal stability [30]. The onset of thermal 221 degradation of PLLA, PLLA-1\% MOF, PLLA-5\% MOF, PLLA-10\% MOF and PLLA222 20\% MOF was observed at 341.6, 330.9, 321.6, 319.6 and $315.2{ }^{\circ} \mathrm{C}$, respectively. The 223 sharp weight loss observed between $300-376^{\circ} \mathrm{C}$ can be ascribed to the thermal degradation 224 of the polymeric chains. Degradation peaks as obtained from derivative profile were 225 observed at $376.6,359.3,354.1,351.2$ and $354.5{ }^{\circ} \mathrm{C}$ for PLLA, PLLA-1\% MOF, PLLA$2265 \%$ MOF, PLLA-10\% MOF and PLLA-20\% MOF, respectively. It was also observed that 227 PLLA-MOF composites weight loss peaked around $2.5 \% .{ }^{\circ} \mathrm{C}^{-1}$ whereas PLLA degradation 228 peaked to $2.35 \% .{ }^{\circ} \mathrm{C}^{-1}$. The decrease in the onset of thermal degradation temperature, 229 decrease in the degradation peak temperature and increase in the weight loss rate as 230 observed in the first derivative profile (Figure $2 b$ ) with the addition of MOF samples can 231 be associated with the MOF particles acting as depoylmerization catalysts, which reduced 232 the thermal degradation activation energy [31-33]. Transition metal based complex 233 compounds including MOF particles have been widely recognised for their catalytic 234 activity due to their partial filled d-orbitals or unsaturated metal sites [34]. Fan et al. [32] 235 studied thermal degradation of PLLA in the presence of either $5 \mathrm{wt} \%$ calcium oxide $(\mathrm{CaO})$ 236 or $5 \mathrm{wt} \%$ magnesium oxide $(\mathrm{MgO})$. Based on the differential thermogravimetric profiles, 
237 they observed that onset of thermal degradation of PLLA dropped from 270 to 180 and $238210^{\circ} \mathrm{C}$ for PLLA-5\% $\mathrm{CaO}$ and PLLA-5\% $\mathrm{MgO}$ systems, respectively. Dai et al [35]

239 fabricated PLA composites with Zn (II) - pyridine 2,5 dicarboxylate MOF. They also

240 observed decreased thermal stability of PLA at elevated tempeartures, which was ascribed

241 to unzipping depolymerization. On the other hand, the degradation of PLA has been

242 reported to be a complex phenomenon due to random scissions leading to linear and cyclic

243 oligomers, which can potentially recombine leading to complex byproducts $[35,36]$.

< Figure 2a and 2b, Table 2>

\subsection{Fourier Transform infrared spectroscopy (FTIR)}

246 To understand the effect of MOF on the PLLA polymeric matrix, PLLA and MOF peaks

247 were superimposed, and peaks which exist only in PLLA matrix were discriminated for 248 further analysis. Figure 3a provides the FTIR spectra of PLLA and PLLA-MIL-53 (Al)

249 MMMs. The FTIR peaks between 2850 and $3000 \mathrm{~cm}^{-1}$ have been assigned to the symmetric 250 and asymmetric stretch of methyl and methylene groups. Medium intensity asymmetric $251 \mathrm{CH}_{3}$ vibration (stretching) and symmetric $-\mathrm{CH}_{3}$ vibration (stretching) have been observed 252 at 2977 and $2952 \mathrm{~cm}^{-1}$. Various factors can significantly impact peak intensity strength of 253 these bands depending on the structure, chain architecture, and packing [37-40]. In 254 addition, orientation, crystallinity and chain restriction can also affect the intensity of 255 various vibrational peaks [41]. We observed a significant decrease in the peak intensity of $256-\mathrm{CH}_{3}$ (asymmetric and symmetric), carbonyl, and $\mathrm{C}-\mathrm{O}-\mathrm{C}$ group vibrations with addition of 2571 wt. \% MOF particles in the PLLA matrix compared to that of the neat PLLA. This 258 decrease can be related to decreased crystallinity and hindered mobility of the PLLA chains 259 due to the strong favorable interfacial interactions between PLLA and MOF particles [1, 
260 42-43]. Yu et al [42] reported decreased FTIR peak intensity for poly(vinylidene fluoride)

261 in 2850 to $3000 \mathrm{~cm}^{-1}$ wavenumber region with the addition of nanoclay. The decreased

262 intensity was ascribed to the strong interaction between polymer and clay platelets which

263 impeded the chain mobility resulting in reduced infrared absorption in this region. The

264 systematically amplified intensity decrease with the increase in the nano-clay content from

2650.2 to $10 \mathrm{wt} \%$ clay also supports the DSC observations.

$<$ Figure 3a $>$

Figure $3 \mathrm{~b}$ shows FTIR spectra around $1750 \mathrm{~cm}^{-1}$ band generally correlated with $268 v(\mathrm{C}=\mathrm{O})$ interchain dipole-dipole interaction. These interchain dipole-dipole interactions 269 depend on the distance between the chains and are influential when the distance between 270 the $\mathrm{C}=\mathrm{O}$ neighboring molecules is less than $2.7 \AA$ [43]. Reduced peak intensity indicates 271 reduced interactions which is supported by the decrease in the crystallinity.

FTIR spectra ranging from wavenumbers $100-800 \mathrm{~cm}^{-1}$ are presented in Figure $3 \mathrm{c}$.

274 Wavenumber $871 \mathrm{~cm}^{-1}$ has been associated to $10_{3}$ helix conformation which has been 275 linked with the alpha crystals in the PLA [37]. The decreased intensity around this 276 wavenumber is also indicative of the reduced crystallinity due to reduced mobility of PLA 277 chains

\subsection{Scanning Electron Microscopy (SEM)}

280 Figure 4 a-f show SEM images of MOF particles, PLLA and various compositions of 281 PLLA-MIL-53 (Al) MMMs. Poor polymer MOF interactions can result in non-selective 
282 interfacial voids. Therefore, a defect free morphology with strong interfacial interaction

283 between filler and polymeric matrix is a precondition to obtaining superior functional

284 performance of these membranes. We observed defect free polymer-filler morphology for

285 PLLA-MIL-53 (Al) MMMs in Figure $4 \mathrm{c}$ to $\mathrm{f}$, which supports our finding of strong 286 interactions between PLLA and MIL-53 (Al) MOF as also supported by DSC and FTIR 287 studies.

$<$ Figure 4 a-f $>$

\section{3.5 Oxygen Permeability}

290 Various industrial or consumer applications such as fresh produce packaging may desire

291 selective and tailored oxygen gas permeability. Controlled oxygen gas permeability may

292 help in extending the shelf life of respiring produce. For oxygen mass transfer $20 \mathrm{wt} \%$

293 MIL-53 (Al) was carefully chosen based on the previous research works its quadrupole

294 moment and polarizability $[3,19,44-45]$. Table 3 shows $\mathrm{O}_{2}$ permeability coefficients of 295 PLLA and PLLA-20\% MIL-53 (Al) MOF. Oxygen permeability coefficient increased by $29626 \%$ with the addition of 20\% Mil-53 (Al) MOF. Improved oxygen gas breathability of 297 these composites can be ascribed to the strong interactions between organic linkers and 298 oxygen gas molecules [19]. 


\section{Conclusions and Future Work}

300 In this study, PLLA, PLLA- 1\% MIL-53(Al), PLLA- 5\% MIL-53(Al), PLLA- 10\% MIL301 53(Al) and PLLA- 20\% MIL-53(Al) MMM films were fabricated using a solvent casting 302 process. DSC, FTIR and SEM data supported good interfacial interactions between PLLA 303 and MIL-53(Al) MOF. Favorable polymer-filler interactions reduced segmental mobility 304 of the chain affecting the crystallinity. Completely amorphous PLLA-MIL-53(Al) MMM 305 films were obtained at $5 \mathrm{wt} . \%$ MOF or above. The presence of MOF increased the rate of 306 degradation of PLLA by de-polymerization reaction as observed in TGA studies. These 307 membranes can easily be fabricated on mass scale by using master-batch and using cast 308 film extrusion provided favorable economics. The future studies can explore the 309 commercialization of these membrane systems.

\section{Acknowledgments}

311 The authors would like to thank funding from Cal Poly State University and Orfalea Mini

312 Summer Support Research Grant. Authors would also like to thank Eric Futak, Jeffrey

313 Norton, Jessie O'Connell, and Nestor Vazquez for help with the experiments. Authors also 314 thank University of Hasselt for allowing Niels Brouwers to study abroad for his graduate 315 research work. Authors are also thankful to IAPRI for awarding Niels Brouwers a 316 scholarship to travel to Cal Poly. 
1. Kathuria A, Abiad MG, and Auras R. Toughening of poly(L-lactic acid) with $\mathrm{Cu}_{3} \mathrm{BTC}_{2}$ metal organic framework crystals. Polymer 2013; 54: 6979-6986.

2. Kathuria A, Al-Ghamdi S, Abiad MG, and Auras R. The influence of $\mathrm{Cu}_{3}(\mathrm{BTC})_{2}$ metal organic framework on the permeability and perm-selectivity of PLLA-MOF mixed matrix membranes. Journal of Applied Polymer Science 2015; 42764 (1-10).

3. Kathuria A, Abiad MG, and Auras R. Deterioration of metal-organic framework crystal structure during fabrication of poly(L-lactic acid) mixed-matrix membranes. Polymer International 2013; 62: 1144-1151.

4. Saiter A, Delpouve N, Dargent E, Oberhauser W, Conzatti L, Cicogna F, Passaglia E. Probing the chain segment mobility at the interface of semi-crystalline polylactide/clay nanocomposites. European Polymer Journal 2016; 274-289.

5. Jiang N, Endoh MK, Koga T, Masui T, Kishimoto H, Nagao M, Satija SK, Taniguchi T. Nanostructures and Dynamics of Macromolecules Bound to Attractive Filler Surfaces. ACS Macro Letters 2015; 4: 838-842.

6. Robertson CG, Lin CJ, Rackaitis M, and Roland CM. Influence of particle size and polymerfiller coupling on viscoelastic glass transition of particle-reinforced polymers. Macromolecules 2008; 41: 2727-2731.

7. Cho SY, Park HH, Yun YS, and Jin H-J. Influence of Cellulose nanofibers on the morphology and physical properties of poly(lactic acid) foaming by supercritical carbon dioxide. Macromolecular Research 2013; 21: 529-533.

8. Mofokeng JP, Luyt AS, Tabi T, and Kovacs J. Comparison of injection moulded natural fibrereinforced composites with PP and PLA as matrices. Journal of Thermoplastic Composite Material 2011, 25: 927-948.

9. Castro-Aguirre E, Iniguez-Franco F, Samsudin H, Fang X, and Auras R. Poly(lactic acid)-Mass production, processing, industrial applications, and end of life. Advanced Drug Delivery Reviews 2016; 107: 333-366.

10. Saeiddlou S, Huneault MA, Li H, and Park CB. Poly(lactic acid) crystallization. Progress in Polymer Science 2012; 37: 1657-1677. Nature 1999; 402: 276-279.

11. Li H, Eddaoudi M, O'Keeffe M, and Yaghi OM. Design and synthesis of an exceptionally stable and highly porous metal-organic framework.

12. Rastogi S, Yao Y, Lippits DR, Höhne GWH, Graf R, Spiess HW, Lemstra PJ. Segmental mobility in the non-crystalline regions of semicrystalline polymers and its implications on melting. Macromolecular Rapid Communication 2009; 30: 826-839.

13. Al-Ghamdi S, Kathuria A, Abiad M and Auras R. Synthesis of nanoporous carbohydrate metalorganic framework and encapsulation of acetaldehyde. Journal of Crystal Growth 2016; 451: 72-78.

14. Lee JY, Farha OK, Roberts J, Scheidt KA, Nguyen ST, and Hupp JT. Metal-organic framework materials as Catalyst. Chemical Society Reviews 2009; 38: 1450-1459.

15. Kreno LE, Leong K, Farha OK, Allendorf M, Duyne RPV, and Hupp JT. Metal-Organic Framework Materials as Chemical Sensors. Chemical Reviews 2012; 
16. Porous metal-organic framework nanoscale carriers as a potential platform for drug delivery and imaging. Nature Materials 2010; 9: 172-178.

17. James SL. Metal-organic frameworks. Chemical Society Reviews 2003; 32: 276-288.

18. Mihaylov M, Chakarova K, Andonova S, Drenchev N, Ivanova E, Pidko EA, Sabetghadam A, Seoane B, Gascon J, Kapteijn F, and Hadjiivanov K. Adsorption of $\mathrm{CO}_{2}$ on MIL-53 (Al): FTIR evidence of the formation of dimeric $\mathrm{CO}_{2}$ species. Chemical Communications 2016; 52: 14941497.

19. Rallapalli P, Prasanth KP, Patil D, Somani RS, Jasra RV, Bajaj HC. Sorption studies of $\mathrm{CO}_{2}$, $\mathrm{CH}_{4}, \mathrm{~N}_{2}, \mathrm{CO}, \mathrm{O}_{2}$ and $\mathrm{Ar}$ on nanoporous aluminum terephthalate [MIL-53(Al)]. Journal of Porous Materials 2011; 18: 205-210.

20. Férey G, Latroche M, Serre C, Millange F, Loiseau T and Percheron-Guégan A. Hydrogen adsorption in the nano-porous metal benzenedicarboxylate $\mathrm{M}(\mathrm{OH})(\mathrm{O} 2 \mathrm{C}-\mathrm{C} 6 \mathrm{H} 4-\mathrm{CO} 2)(\mathrm{M}=$ Al3+, Cr3+), MIL-53. Chemical Communications 2003; 2976-2977.

21. Bourrelly S, Llewellyn PL, Serre C, Millange F, Loiseau T, and Férey G. Different Adsorption Behaviors of Methane and Carbon Dioxide in the Isotypic Nanoporous Metal Terephthalates MIL-53 and MIL-47

22. Vinh-Thang H, and Kaliaguine S. Predictive models for mixed-matrix membrane performance: A review; Chemical Review 2013; 113:4980-5028.

23. Pan P Zhu B and Inoue Y. Ethalpy relaxation and embrittlement of poly(L-lactide) during physical aging. Macromolecules 2007; 40: 9664-9671.

24. Cangialosi D, Boucher VM, Alegria A, and Colmenero J. Physical aging in polymers and polymer nanocomposites: recent results and open questions. Soft Matter 2013; 9: 8619-8630.

25. Napolitano S, and Wubbenhorst $M$. The life time of the deviations from bulk behavior in polymers confined at the nanoscale. Nature Communications 2011; 2: 260.

26. Huang HD, Ren PG, Xu JZ, Xu L, Zhong GJ, Hsiao BS, and Li ZM. Improved barrier properties of poly(lactic acid) with randomly dispersed graphene oxide nanosheets. Journal of Membrane Science 2014; 464: 110-118.

27. Dorgan J R, Rheology of Poly(lactic acid). Poly(Lactic Acid): Synthesis, Structures, Properties, Processing, and Applications, Edited by Auras R, Lim L, Selke S and Tsuji H, Wiley Series Published 2010; Chapter 10: 125-139.

28. Gagliradi S, Arrighia V, Ferguson R and Telling MTF. Restricted dynamics in polymer-filler systems. Physica B 2001; 301: 110-114.

29. Fragiadakis D, Bokobza L, and Pissis P. Dynamics near the filler surface in natural rubbersilica nanocomposites. Polymer 2011; 52: 3175-3182.

30. Loiseau T, Serre C, Huguenard C, Fink G, Taulelle F, Henry M, and bat A rationale for the large breathing of the porous Aluminum Terephthalate (MIL-53) upon hydration.

31. Motoyama T, Tsukegi T, Shirai Y, Nishida H, and Endo T. Effects of $\mathrm{MgO}$ catalyst on depolymerization of poly-L-lactic acid to L,L-lactide. Polymer degradation and stability 2007; 92: $1350-1358$.

32. Fan Y, Nishida H, Mori T, Shirai Y, and Endo T. Thermal degradation of poly(L-lactide): effect of alkali earth metal oxides for selective L, L-lactide formation. Polymer 2004; 45: 1197-1205. 
33. Tsuji $\mathrm{H}$ and Fukui I. Enhanced thermal stability of poly(lactide)s in the mely by enantiomeric polymer blending. Polymer 2003; 10: 2891-2896.

34. Yoon JW, Seo Y-K, Hwang YK, Chang J-S, Leclerc H, Wuttke S, Bazin P, Vimont A, Daturi M, Bloch E, Llewellyn PL, Serre C, Horcajada P, Greneche J-M, Rodrigues AE, Ferey G. Controlled Reducibility of a Metal-Organic Framework with Coordinatively unsaturated sites for preferential gas sorption. Angewandte Chemie 2010; 122: 6085-6088.

35. Dai X, Cao Y, Shi X, and Wang X. Non-isothermal crystallization kinetics, thermal degradation behavior and mechanical properties of poly(lactic acid)/MOF composites prepared by meltblending methods. RSC Advances 2016; 2046-2069.

36. Yu H, Huang N, Wang $\mathrm{C}$ and Tang Z. Modeling of poly(L-lactide) thermal degradation: theoretical prediction of molecular weight and polydispersity index. Journal of Applied Polymer Science 2003; 88: 2557-2562.

37. Poly(Lactic Acid): Synthesis, Structures, Properties, Processing, and Applications, Edited by Auras R, Lim L, Selke S and Tsuji H, Wiley Series Published 2010; Chapter 8: 97-112.

38. Robinson JW, Frame EMS, Frame II GM. Undergraduate Instrumental Analysis, Sixth Edition, MARCEL DEKKER, NY.

39. Verma SP, Wallach DFH. Raman spectra of some saturated, unsaturated and deuterated $\mathrm{C}_{18}$ fatty acids in the $\mathrm{HCH}$-deformation and $\mathrm{CH}$-stretching regions.

40. Messmer MC, Conboy JC, and Richmond GL. Observation of molecular ordering at the liquidliquid interface by resonant sum frequency generation. Journal of the American Chemical Society 1995; 17: 8039-8040.

41. Paragkumar NT, Edith D and Six JL. Surface characteristics of PLA and PLGA films. Applied Surface Science 253: 5; 2758-2764.

42. $\mathrm{Yu} \mathrm{L}$ and Cebe P. Crystal polymorphism in electrospun composite nanofibers of poly(vinylidene fluoride) with nanoclay. Polymer 2009; 50: 2133-2141.

43. Zhang J, Tsuji H, Noda I, and Ozaki Y. Structural changes and crystallization dynamics of Poly(L-lactide) during the cold-crystallization process investigated by infrared and twodimensional infra-red correlation spectroscopy. Macromolecules 2004; 37: 6433-6439.

44. Adams R, Carson C, Ward J, Tannenbaum R, Koros W. Metal organic framework mixed matrix membranes for gas separations. Microporous and Mesoporous Materials 2010; 131: 13-20.

45. Elangovan D, Nidoni U, Yuzay I E, Selke SEM and Auras R. Industrial and Engineering Chemistry Research 2011; 50: 11136-42. 
432 Table 1. Thermal characteristics of neat PLLA and PLLA-MIL-53 (Al) MMM films

433 derived from the $2^{\text {nd }}$ heating cycle

434 Table 2. Thermo-gravimetric analysis of neat PLLA and PLLA-MOF MMM films

435 Table 3. $\mathrm{O}_{2}$ permeability coefficient of PLLA and PLLA-20\% MIL-53 (Al) MOF MMM

436 films

$438 \quad$ List of Figures

439 Figure 1. DSC thermograms of PLLA and PLLA-MIL-53 (Al) MMM films derived from 440 the $2^{\text {nd }}$ heating cycle.

441 Figure 2a. TGA of PLLA, MIL-53 (Al) and various PLLA-MIL-53 (Al) MMM films.

442 Figure 2b. First derivative weight change as a function of temperature.

443 Figure 3a. FTIR Spectra of PLLA and PLLA- MIL-53 (Al) MMM films from wavenumber $444 \quad 3000-2800 \mathrm{~cm}^{-1}$.

445 Figure 3b. FTIR Spectra of PLLA and PLLA-MIL-53 (Al) MMM films from wavenumber $446 \quad 1650-1850 \mathrm{~cm}^{-1}$.

447 Figure 3c. FTIR Spectra of PLLA and PLLA-MIL-53 (Al) MMM films from wavenumber $448 \quad 950-800 \mathrm{~cm}^{-1}$.

449 Figure 4. a). SEM image of MIL-53 (Al), scale size $50 \mu \mathrm{m}$ b). SEM image of PLLA, scale 450 size $20 \mu \mathrm{m} \mathrm{c}$ ). SEM image of PLLA-1\% MIL-53 (Al), scale size $20 \mu \mathrm{m}$. d). SEM image 451 of PLLA-5\% MIL-53 (Al), scale size $20 \mu \mathrm{m}$. e). SEM image of PLLA-10\% MIL-53 (Al), 452 scale size $20 \mu \mathrm{m}$. f). SEM image of PLLA-20\% MIL-53 (Al), scale size $20 \mu \mathrm{m}$. 
Table 1. Thermal characteristics of neat PLLA and PLLA-MIL-53 (Al) MMM films derived from the $2^{\text {nd }}$ heating cycle.

\begin{tabular}{|c|c|c|c|c|c|c|}
\hline \multirow{2}{*}{ Sample } & \multirow[t]{2}{*}{$T_{g}\left({ }^{\circ} \mathrm{C}\right)$} & \multicolumn{2}{|c|}{ Cold Crystallization } & \multicolumn{3}{|c|}{ Melting } \\
\hline & & $T_{c o}\left({ }^{\circ} \mathrm{C}\right)$ & $\Delta H_{c}(\mathrm{~J} / \mathrm{g})$ & $T_{m}\left({ }^{\circ} \mathrm{C}\right)$ & $\Delta H_{m}(\mathrm{~J} / \mathrm{g})$ & $X c(\%)$ \\
\hline PLLA & $56.6 \pm 0.5^{\mathrm{A}}$ & $105.1 \pm 1.2^{\mathrm{A}}$ & $8.7 \pm 1.9^{\mathrm{A}}$ & $148.3 \pm 0.4^{\mathbf{A}}$ & $11.7 \pm 2.2^{\mathrm{A}}$ & $3.2 \pm 0.1^{\mathrm{A}}$ \\
\hline PLLA- $1 \%$ MOF & $59.0 \pm 0.5^{\mathbf{B}}$ & $108.6 \pm 4.2^{\mathbf{A}}$ & $2.7 \pm 2.6^{\mathbf{B}}$ & $149.0 \pm 0.2^{\mathbf{A}, \mathbf{B}}$ & $4.7 \pm 2.8^{\mathbf{B}}$ & $3.1 \pm 0.0^{\mathrm{A}}$ \\
\hline PLLA-5\% MOF & $57.9 \pm 0.3^{\mathrm{C}}$ & - & - & $149.4 \pm 0.4^{\mathbf{B}}$ & $0.5 \pm 0.1^{\mathrm{C}}$ & $0.5 \pm 0.1^{\mathbf{B}}$ \\
\hline $\begin{array}{l}\text { PLLA-10\% } \\
\text { MOF }\end{array}$ & $57.3 \pm 1.4^{\mathbf{A}, \mathbf{B}, \mathbf{C}}$ & - & - & - & - & - \\
\hline $\begin{array}{l}\text { PLLA-20\% } \\
\text { MOF }\end{array}$ & $60.1 \pm 0.6^{\mathbf{B}}$ & - & - & - & - & - \\
\hline
\end{tabular}
denotes no results were obtained due to absence of cold crystallization and melting peaks. 
459 Table 2. Thermo-gravimetric analysis of neat PLLA and PLLA-MOF MMM films.

\begin{tabular}{|c|c|c|c|c|}
\hline \multirow{2}{*}{ Sample } & \multirow{2}{*}{$\begin{array}{c}\begin{array}{c}\text { Onset } \\
\text { Thermal } \\
\text { degradation }\end{array} \\
\left({ }^{\circ} \mathrm{C}\right)\end{array}$} & \multicolumn{2}{|c|}{ Weight loss peaks } & \multirow{2}{*}{$\begin{array}{c}\begin{array}{c}\text { Peak derivative } \\
\text { weight loss }\end{array} \\
\left(\% /{ }^{\circ} \mathrm{C}\right)\end{array}$} \\
\hline & & Peak $1\left({ }^{\circ} \mathrm{C}\right)$ & Peak $2\left({ }^{\circ} \mathrm{C}\right)$ & \\
\hline PLLA & $341.6 \pm 2.0^{\mathbf{A}}$ & $136.6 \pm 2.0^{\mathbf{A}}$ & $376.6 \pm 1.4^{\mathrm{A}}$ & $2.35 \pm 0.02^{\mathrm{A}}$ \\
\hline PLLA- $1 \%$ MOF & $330.9 \pm 0.8^{\mathbf{B}}$ & $136.5 \pm 0.9^{\mathbf{A}}$ & $359.3 \pm 0.8^{\mathrm{B}}$ & $2.50 \pm 0.03^{\mathrm{A}, \mathrm{B}}$ \\
\hline PLLA-5\% MOF & $321.6 \pm 1.3^{\mathrm{C}}$ & $135.7 \pm 0.8^{\mathbf{A}}$ & $354.1 \pm 0.6^{\mathrm{C}, \mathrm{D}}$ & $2.55 \pm 0.06^{\mathrm{B}}$ \\
\hline PLLA-10\% MOF & $319.6 \pm 0.9^{\mathrm{C}}$ & $134.6 \pm 1.4^{\mathrm{A}}$ & $351.2 \pm 0.9^{\mathrm{D}}$ & $2.52 \pm 014^{\mathrm{A}, \mathrm{B}}$ \\
\hline PLLA-20\% MOF & $315.2 \pm 0.9^{\mathrm{C}}$ & $133.3 \pm 2.0^{\mathbf{A}}$ & $354.5 \pm 1.5^{\mathrm{C}}$ & $2.51 \pm 0.01 \mathrm{~A}, \mathrm{~B}$ \\
\hline
\end{tabular}

460 Note: Values in the same column with same capital superscript letters are not statistically

461 significantly different at $\alpha=0.05$. The "-"symbol denotes no results were obtained due to absence 462 of cold crystallization and melting peaks.

463

464 Table 3. $\mathrm{O}_{2}$ permeability coefficient of PLLA and PLLA-20\% MIL-53 (Al) MOF MMM 465 films.

$\mathrm{O}_{2}$ Permeability Coefficient x $10^{-18}\left(\mathrm{~kg} \cdot \mathrm{m} \cdot \mathrm{m}^{-2} \cdot \mathrm{s}^{-1} \cdot \mathrm{Pa}^{-1}\right)$

\begin{tabular}{lc}
\hline PLLA & $2.27 \pm 0.09^{\mathrm{A}}$ \\
PLLA-20\% MOF & $2.87 \pm 0.34^{\mathrm{B}}$
\end{tabular}




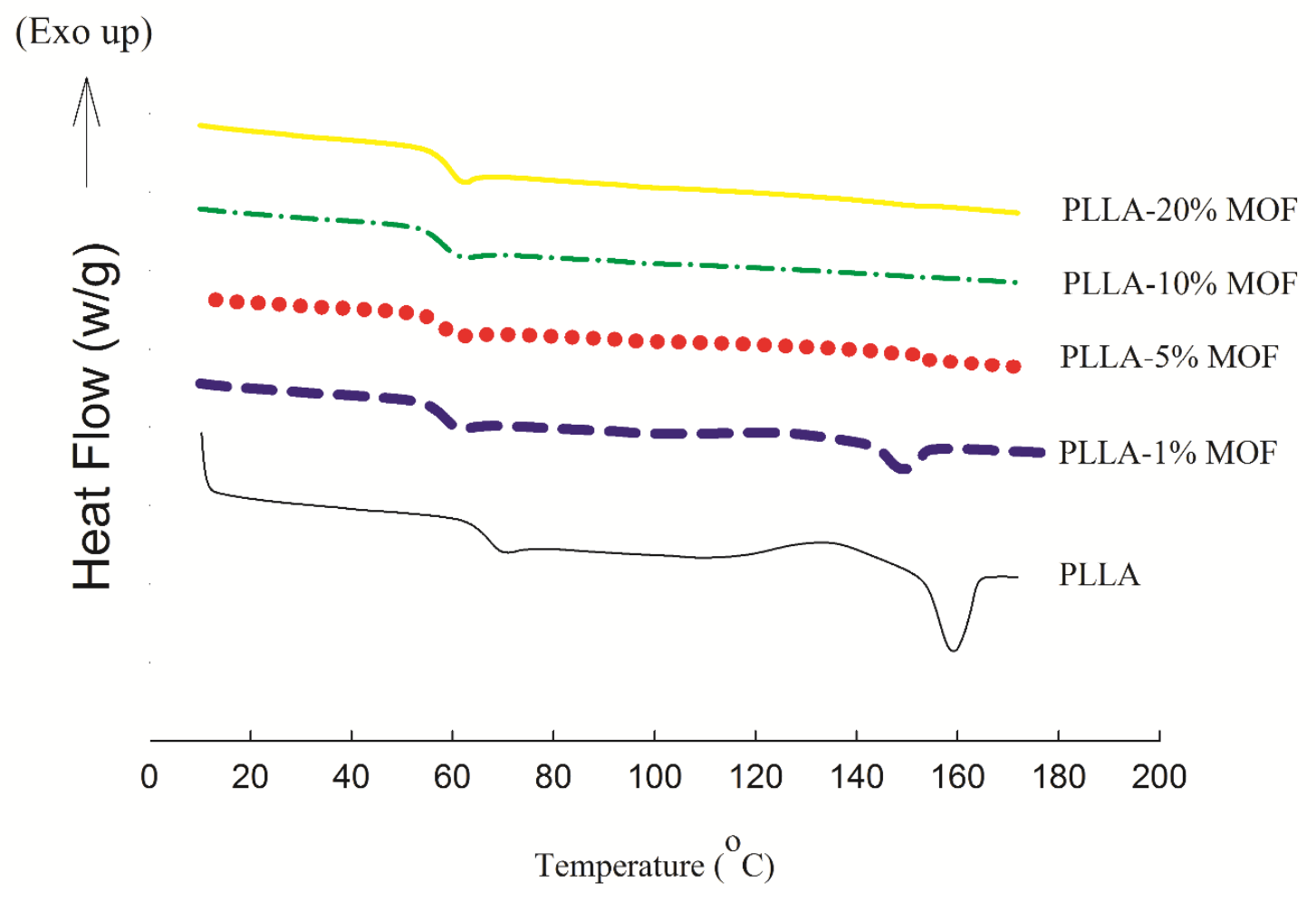

469 Figure 1. DSC thermograms of PLLA and PLLA-MIL-53 (Al) MMM films derived from 470 the $2^{\text {nd }}$ heating cycle. 


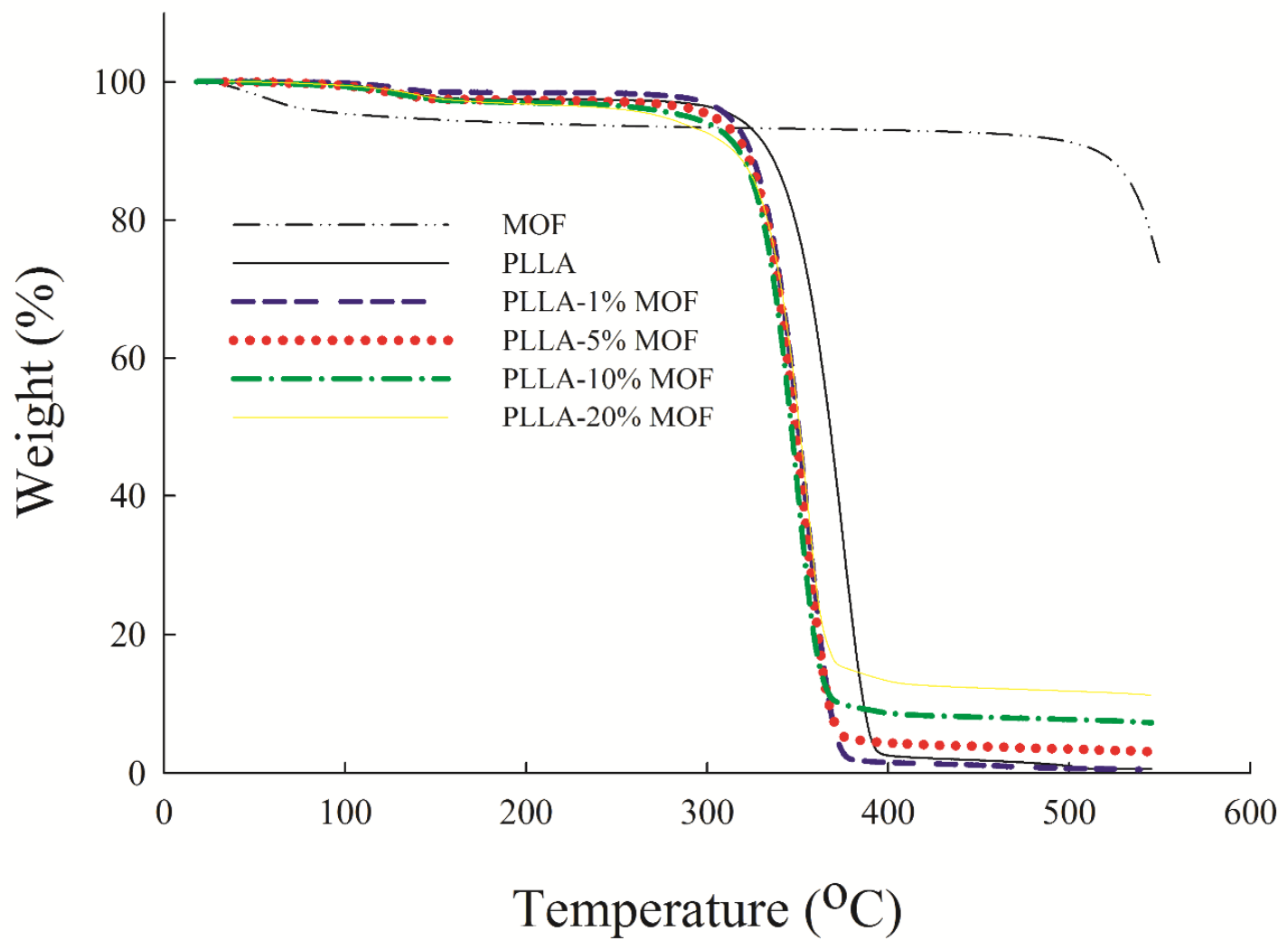

Figure 2a. TGA of PLLA, MIL-53 (Al) and various PLLA-MIL-53 (Al) MMM films. 


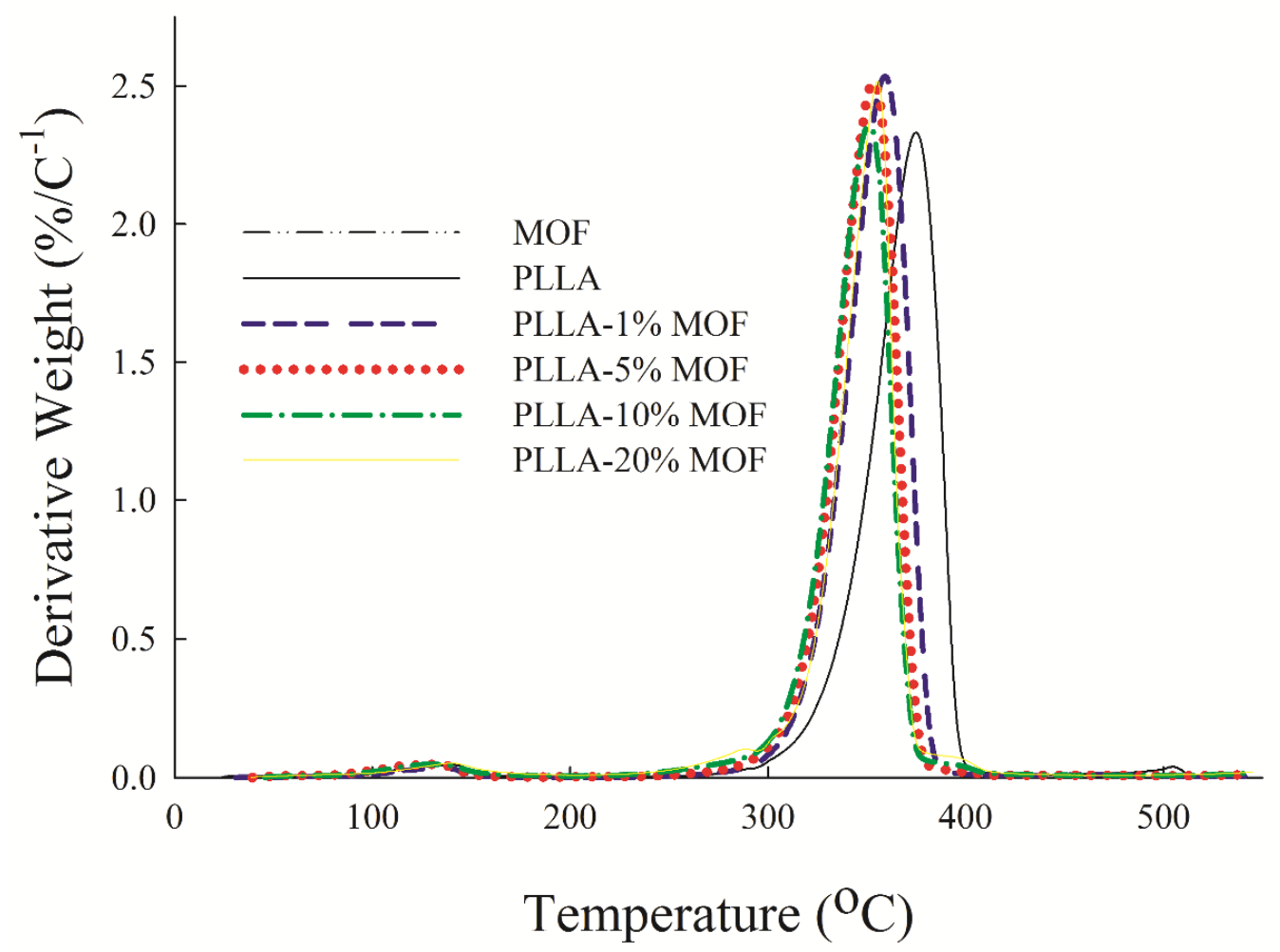

474

Figure 2b. First derivative weight change as a function of temperature. 
a.

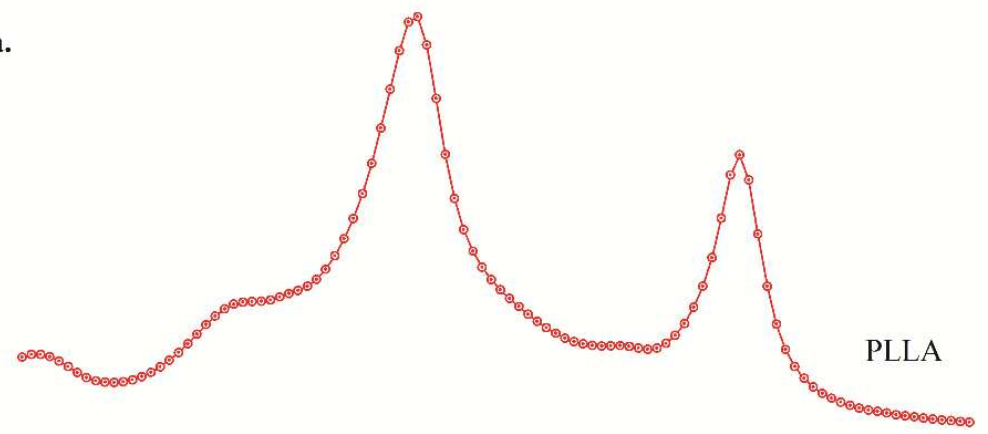

b.

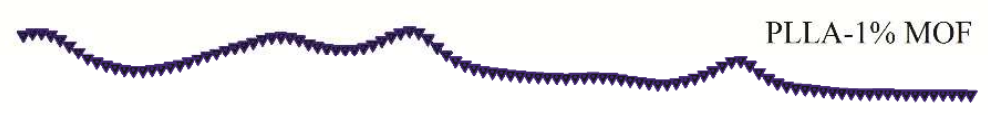

c.

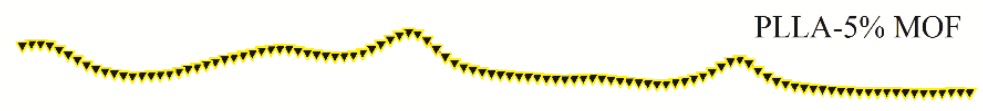

d.

PLLA-10\% MOF

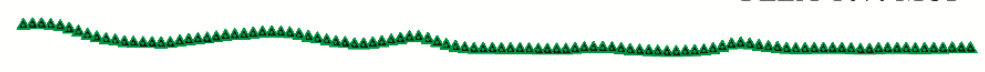

e.

PLLA-20\% MOF

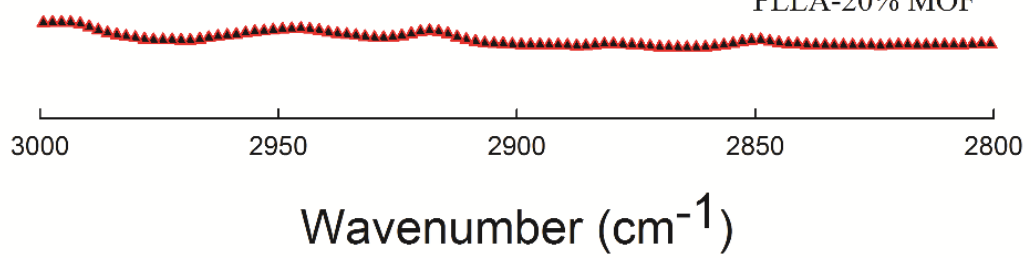

475

Figure 3a. FTIR Spectra of PLLA and PLLA- MIL-53 (Al) MMM films from wavenumber $477 \quad 3000-2800 \mathrm{~cm}^{-1}$. 

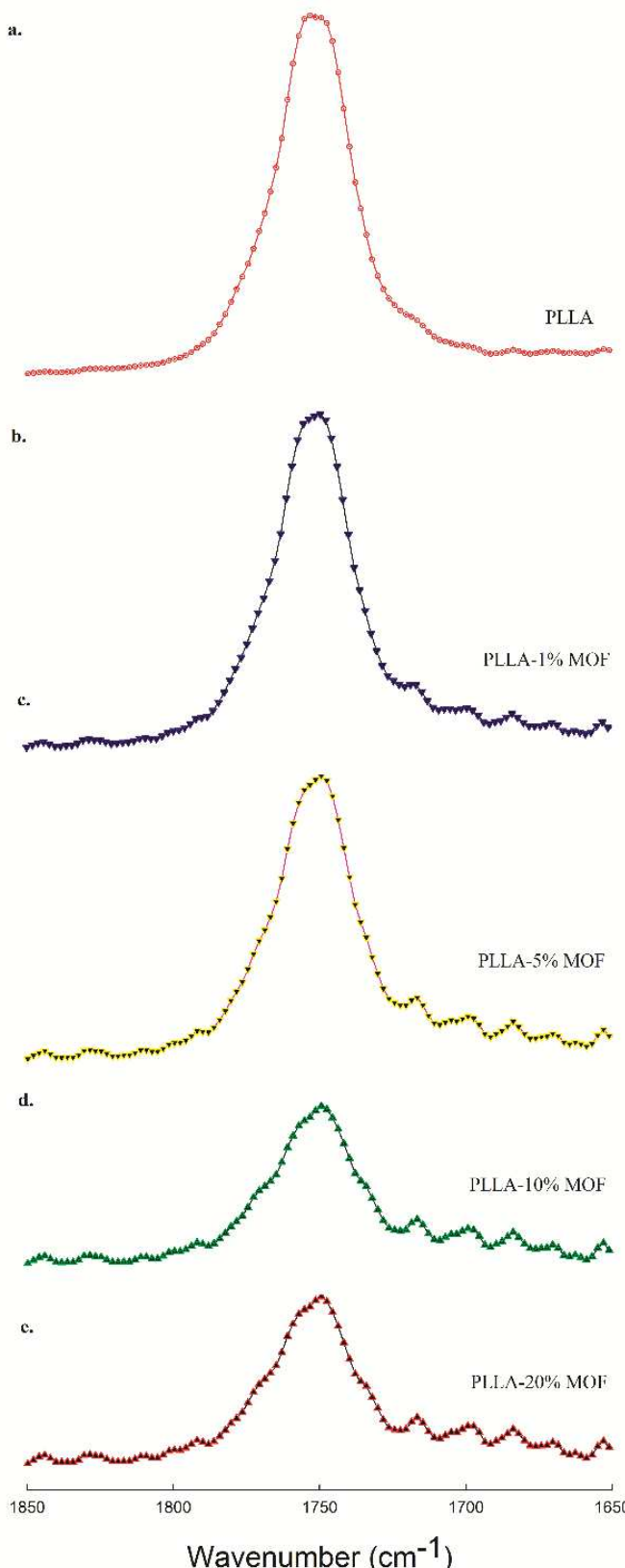

Wavenumber $\left(\mathrm{cm}^{-1}\right)$

479 Figure 3b. FTIR Spectra of PLLA and PLLA-MIL-53 (Al) MMM films from wavenumber $480 \quad 1650-1850 \mathrm{~cm}^{-1}$. 

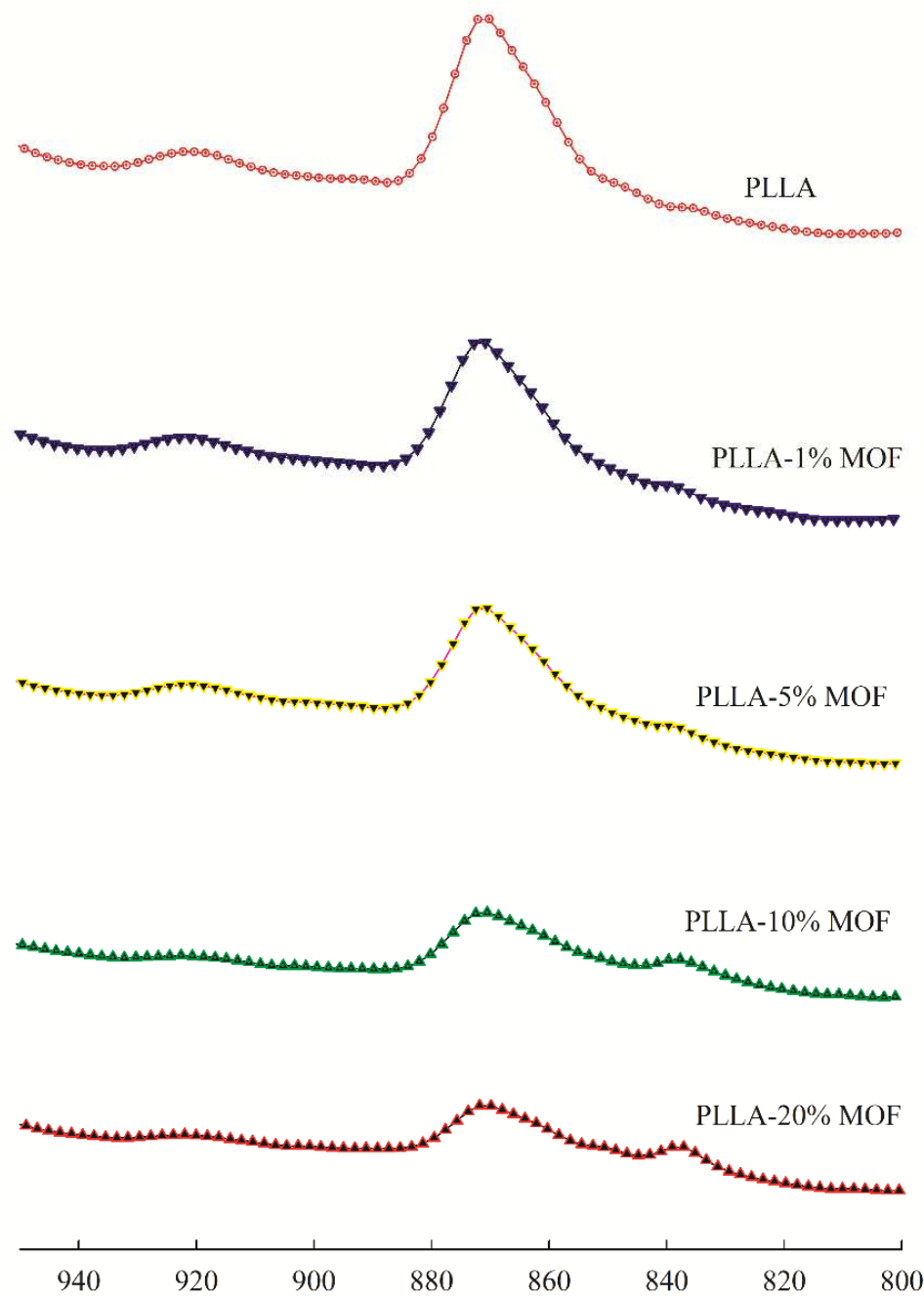

Wavenumber $\left(\mathrm{cm}^{-1}\right)$

482 Figure 3c. FTIR Spectra of PLLA and PLLA-MIL-53 (Al) MMM films from wavenumber $483950-800 \mathrm{~cm}^{-1}$. 
484
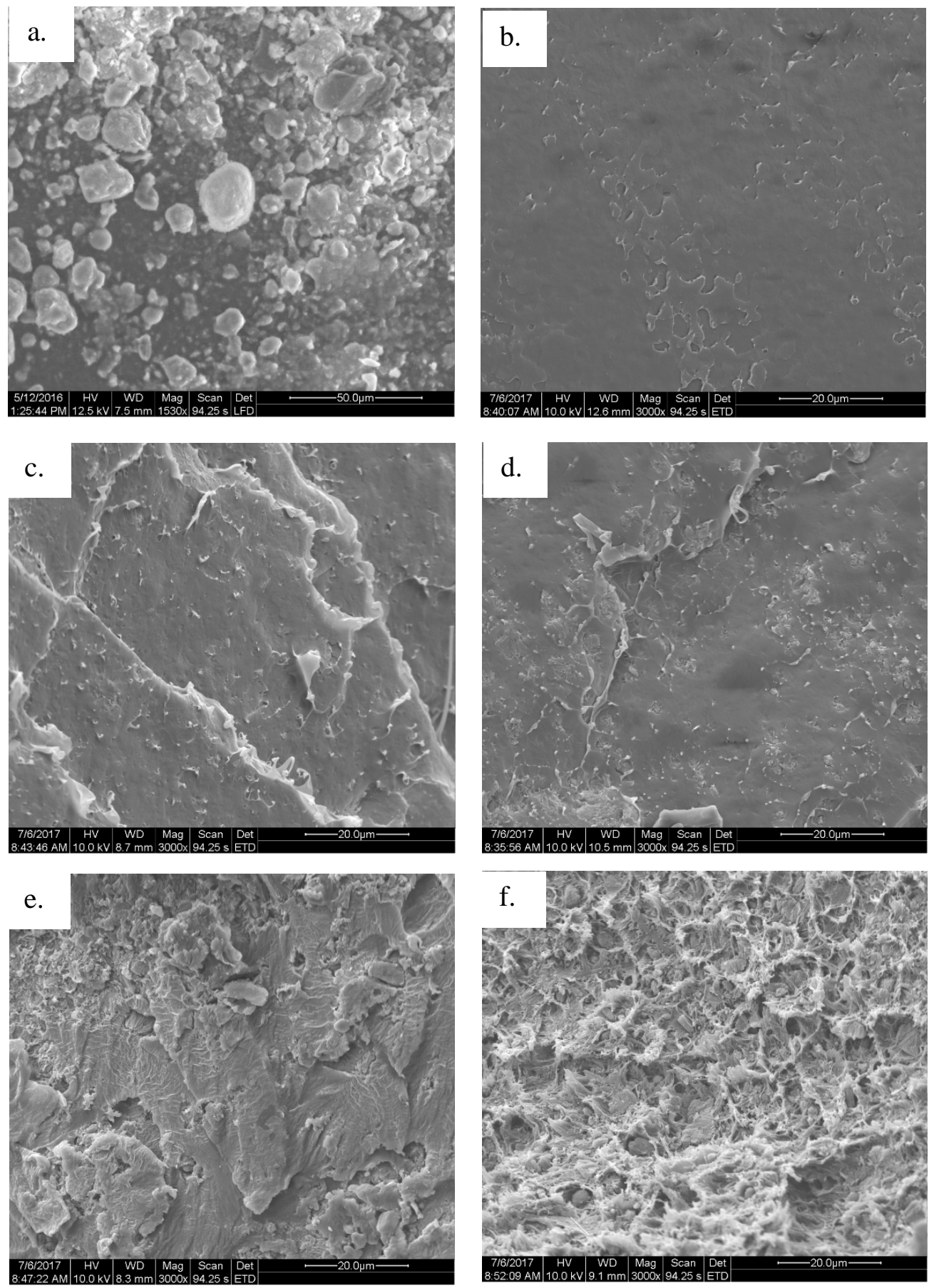

485

486 Figure 4. a). SEM image of MIL-53 (Al), scale size $50 \mu \mathrm{m} \mathrm{b})$. SEM image of PLLA, scale 487 size $20 \mu \mathrm{m} \mathrm{c}$ ). SEM image of PLLA-1\% MIL-53 (Al), scale size $20 \mu \mathrm{m}$. d). SEM image 488 of PLLA-5\% MIL-53 (Al), scale size $20 \mu \mathrm{m}$. e). SEM image of PLLA-10\% MIL-53 (Al), 489 scale size $20 \mu \mathrm{m}$. f). SEM image of PLLA-20\% MIL-53 (Al), scale size $20 \mu \mathrm{m}$. 